\title{
A Novel Plantar Stimulation Technology for Improving Protective Sensation and Postural Control in Patients with Diabetic Peripheral Neuropathy: A Double-Blinded, Randomized Study
}

\author{
Bijan Najafia, ${ }^{a}$ Ryan T. Crews ${ }^{a}$ James S. Wrobel ${ }^{a, c}$ \\ ${ }^{a}$ CLEAR, Scholl College of Podiatric Medicine at Rosalind Franklin University of Medicine and Science, \\ North Chicago, III., b Southern Arizona Limb Salvage Alliance (SALSA), University of Arizona College of Medicine, \\ Tucson, Ariz., and ' University of Michigan, Medical School, Ann Arbor, Mich., USA
}

\section{Key Words}

Diabetes · Diabetic foot · Diabetic peripheral neuropathy · Balance · Electrical stimulation - Randomized control trial . Fall prevention

\begin{abstract}
Background: Many diabetes-related peripheral neuropathy (DPN) patients also experience postural instability. Objective: This study examined the effect of electrical stimulation therapy on DPN patients' postural control as well as recovery of plantar sensation. Methods: 54 patients with mild to moderate DPN were enrolled into this study evaluating treatment of DPN with electrical stimulation provided via aqueous solution. The subjects were randomized into either sham or active stimulation. Treatments were administered 5 times per week for 6 weeks. Changes in neuropathy severity were assessed via Semmes-Weinstein monofilament tests and vibration perception threshold (VPT) tests. The potential benefit in postural control was assessed in a subsample of 13 subjects by measuring the area of center of mass (COM) sway. Results: The active group saw a significant $(p<0.05)$ improvement in VPT at treatment week 6 in comparison to the sham group. The difference did not remain significant at follow-up visits. There were no significant differences between groups for the Semmes-Weinstein monofilament test.
\end{abstract}

However, time was a significant main effect, with both groups improving over the course of the study. The area of COM sway was significantly reduced on average by $36 \%$ at treatment week 2 compared to the baseline. Although at treatment week 6 an additional 7\% reduction in COM sway was observed compared to treatment week 2 , the improvement from treatment week 2 to treatment week 6 was not significant ( $p>0.6)$. Interestingly, no significant difference was observed in the sham group during the study $(p>0.7)$. Conclusion: This randomized pilot study provides preliminary data on the potential of electrical stimulation via aqueous solution to improve protective sensation and postural stability in DPN patients.

C 2013 S. Karger AG, Basel

\section{Background}

Diabetic peripheral neuropathy (DPN) is prevalent among $50 \%$ of patients with 20 years of diabetes, making this the most common symptomatic complication particularly among older adults $[1,2]$. People with DPN frequent-

Abstracts from this work were presented at the American Diabetes Association's Scientific Sessions and The Diabetic Foot, Noordwijkerhout, The Netherlands.

\section{KARGER}

E-Mail karger@karger.com

www.karger.com/ger
(C) 2013 S. Karger AG, Basel

0304-324X/13/0595-0473\$38.00/0
Bijan Najafi, $\mathrm{PhD}$

Interdisciplinary Consortium for Advanced Motion Performance (iCAMP) Southern Arizona Limb Salvage Alliance (SALSA), Arizona Center on Aging University of Arizona College of Medicine, Tucson, AZ 85724-5072 (USA)

E-Mail najafi.bijan@gmail.com 
ly suffer from concomitant postural instability that can lead to falls, fracture, depression, anxiety, and decreased quality of life [3-6]. Large cohort studies of older individuals at risk for falls have reported an increased risk with diabetes $[7,8]$. In the Women's Health and Aging Study of 1,002 women, Volpato et al. [8] reported diabetes status demonstrated a $44 \%$ increased risk of falls over 3 years in their multivariate model. In the study of osteoporotic fractures $(n=9,249)$, Schwartz et al. [7] reported a $68 \%$ increased multiple falls risk in diabetics over 2 years. Incident falls are also increased in patients with previous foot ulceration [9].

Interestingly, poor balance appears to describe more of the fall risk association than loss of sensation or decreased vibratory perception [7]. Other authors have also described loss of sensation falling out of a multivariate model for conservative gait patterns in persons with diabetes [10]. Schwartz et al. [7] reported poor balance as assessed by tandem gait and standing described 23 and $14 \%$ of the fall risk association compared with 3 and $6 \%$ for monofilament insensitivity and decreased vibration perception.

Peripherally insensate neuropathy $(\mathrm{PN})$, defined as one or more insensate areas in either foot, is also prevalent among nondiabetic older adults. Centers for Disease Control and Prevention (CDC) analyzed data collected during 1999-2002 from the National Health and Nutrition Examination Survey (NHANES) and reported that approximately $12.9 \%$ of US adults aged 40 years or older had PN with one fourth having severe PN [11]. The same report suggested that among adults aged 40 years or greater, the prevalence of lower extremity diseases was higher among persons aged 75 years or older (40.8\%) and 60-74 years (26.2\%) than among persons aged $40-59$ (12.3\%). These data suggest that lower extremity disease and lack of foot sensation or peripheral neuropathy are common among older adults and should receive more attention, particularly when fall prevention is addressed among older adults.

Recently, a large body of basic science and clinical studies have shown conclusively that low-level mechanical or electrical noise presented directly to sensory neurons can significantly enhance their ability to detect weak signals [12-18]. This phenomenon of noise improving or stochastic resonance is resulting from an effect of noise on information transfer and processing. More specifically, in nonlinear systems that involve a threshold (e.g. sensory neurons), low-level noise can be added to subthreshold signals, thus producing a suprathreshold signal which helps to sensitize the system so that small signals or stimuli, which in a no-noise environment would not be discernible, are now apparent [18]. Therefore, it stands to reason that electrical stimulation applied to the feet could improve balance based on an increased sensitivity to somatosensory signals from the foot, thereby reducing falls attributed to decreased tactile sensitivity and awareness of the foot's orientation and position.

One study suggested that mechanical noise stimulation improves vibration and tactile perception in diabetic patients with moderate to severe neuropathy while the vibration is occurring [18]. However, we found no studies that explored whether there are poststimulation benefits of stimulation treatment modalities for individuals with DPN. The purpose of this pilot study was to test whether an aqueous form of transcutaneous low-level electrical stimulation treatment for DPN patients may provide a lasting improvement in protective sensation and postural control that is evident when the treatment is not actively occurring.

\section{Research Design and Methods}

Subjects

Fifty-four subjects with type 1 or type 2 diabetes and mild to moderate DPN were enrolled at four clinical centers. Mild to moderate DPN was defined as insensitivity of a $10 \mathrm{~g}$ monofilament at 1-3 sites in the following locations: hallux, 1st, 3rd, and 5th metatarsal heads [19]. For both feet of a subject to be treated, they were required to meet the monofilament requirement. If only one foot was eligible, only that one received study treatment. Patients with clinically detectable peripheral arterial disease, active ulcer, implanted electrical medical device, cardiac arrhythmia, epilepsy, other seizure disorder, medical condition sensitive to electrical disturbance, or inability to walk $100 \mathrm{ft}$ were excluded. Eligible and willing patients signed a local institutional review board approved informed consent prior to screening.

\section{Protocol}

The study consisted of a 6-week treatment phase of 5 treatments per week. During the treatment phase, patients sat while soaking their feet for $30 \mathrm{~min}$ in a footbath (WaveRx ${ }^{\mathrm{TM}}$; fig. 1) containing two foot wells each with two electrode plates connected to an electrical stimulator. Patients were instructed at the initiation of each treatment to gradually increase the stimulator's power to $40 \%$ or until the patient felt a comfortable tingling sensation (it should be noted, however, that none of the patients felt stimulation prior to reaching $40 \%$ ). Patients were randomized to receive active or sham stimulators in a 1:1 ratio. If the device was active it would deliver $120-\mathrm{Hz}$ pulsed current waveforms up to a maximum of 50 $\mathrm{mA}$. The active group received stimulation, while the stimulator was nonfunctional in the sham group. The devices were identical in appearance and both study personnel and subjects were blinded to which units were active. All participants were evaluated for changes in plantar sensation, vibratory perception testing, and potential adverse events. During the treatment phase of the study, subjects returned to clinic for study visits every 2 weeks. Six weeks after completing the treatment phase, subjects returned for a follow-up visit. A subset of 20 subjects completed a secondary followup visit 12 weeks after completing treatment. None of the outcome 
Fig. 1. Electrical stimulation footbath (WaveRx, Inc., Waltham, Mass., USA).

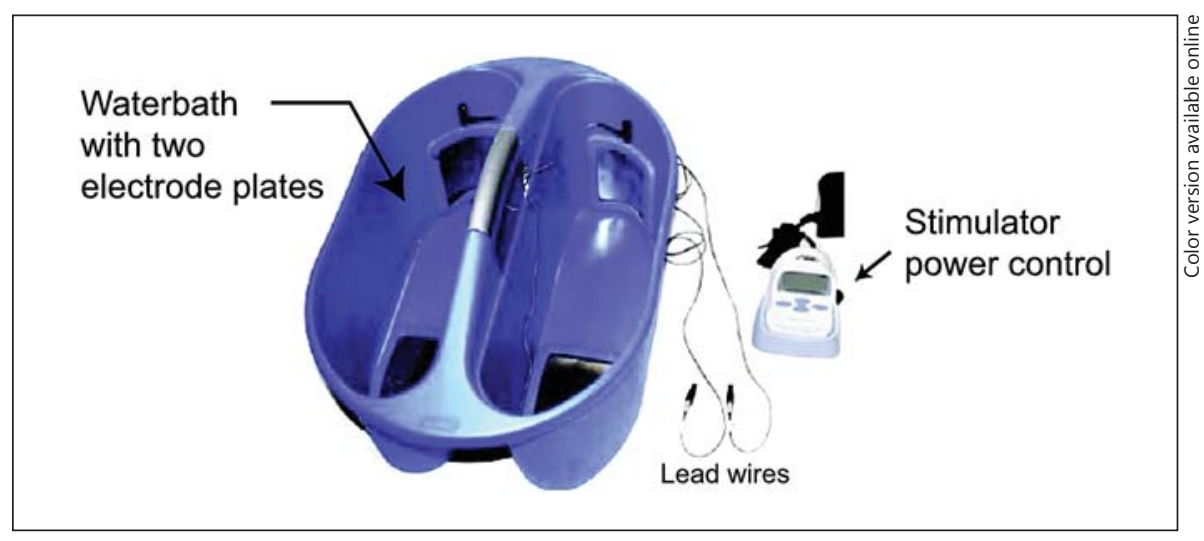

assessments were conducted while receiving active or sham stimulation. The first treatment occurred in the clinic after the collection of baseline data. The only other treatment session conducted in conjunction with a study visit was the treatment that coincided with the first follow-up visit. The administration of treatment at the first follow-up visit was done after outcome assessments were completed. Treatment was conducted at the visit in order to allow an investigator to observe and confirm the participants were following the protocol correctly.

\section{Outcome Assessments}

Subjects' tactile sensation was assessed via monofilaments at the 4 sites on the plantar surface of the foot used for screening. However, for outcome evaluation, testing was conducted at each site with each of the following grades of monofilaments: 2, 4, 6, 8, 10 , and $15 \mathrm{~g}$. For each site and monofilament grade pairing, the monofilament was pressed to the skin until the point of buckling 3 times. After all monofilament tests were completed for a visit, each foot's total score was calculated with a range of 0-72 detections. Subjects' vibration perception threshold (VPT) was assessed at the distal aspect of the great toe as described elsewhere [20,21]. With eyes shut in a seated position, subjects were asked to identify when they perceived a vibratory sensation on the great toe (VPT meter; Xilas Medical, San Antonio, Tex., USA). The value was treated as a continuous variable within the range of 1-100 V. Change scores were calculated for both the monofilament and VPT scores for each treatment and follow-up visit relative to baseline. If a patient received study treatment for both feet, the mean value for both feet was used for analyses.

From the original study, 13 subjects were assessed for postural control. Two wearable sensors (BalanSens ${ }^{\mathrm{TM}}$; Biosensics LLC, Cambridge, Mass., USA) each including a triaxial accelerometer, triaxial gyroscope and a triaxial magnetometer were attached to subject's shin and lower back to estimate 3D angles of the hip and ankle. Using anthropometric data of each subject (e.g. body mass and height), the position of each link's center of mass (COM) in the anterior-posterior and medial-lateral directions were estimated [4]. Postural control was assessed at baseline and every 2 weeks during the treatment phase and 6 months later. Postural control was quantified by measuring the area of COM sway. Balance was examined according to Romberg's protocol for the duration of $30 \mathrm{~s}$ [4].

Electrical Stimulation Therapy for Improving DPN
Statistical Analysis

The monofilament and VPT change scores (with the exception of 12-week follow-up scores) were each analyzed by two-way (treatment and visit) repeated-measures ANOVA. The Kolmogorov-Smirnov test was used for testing normal distribution. Post hoc analyses for significant $(\mathrm{p}<0.05)$ main effects or interactions were done using a Sidak adjustment if more than two values were compared, or by independent $t$ tests if only two values were compared. In order to avoid excessive replacement scores at the 12week follow-up visit (only the subset of 20 subjects completed this visit) these scores were analyzed by independent $t$ tests independently of the repeated-measures ANOVA. Independent-sample Kruskal-Wallis one-way ANOVA tests were used to examine the difference between groups.

Repeated-measures ANOVA test was used to examine the significant change in balance during the 6-week treatment phase and retention during follow-up phase for each group. When a significant difference $(\mathrm{p}<0.05)$ was found, the Student-Newman-Keuls correction was used as the post hoc to assess pairwise comparisons. Spearman correlation of coefficient was used to examine correlation between balance parameters and patient clinical data such as $\mathrm{HbA}_{1 \mathrm{C}}$ level and patient sensitivity to the VPT test. Results were expressed as means \pm SD. Missing monofilament or VPT or balance values for follow-up visits were replaced by the mean score of the subject's other follow-up visits. A p value $\leq 0.05$ was considered statistically significant. The person who analyzed the data was blind to the type of intervention. Statistical analyses were performed using SPSS $^{\circledR}$ version 19 (SPSS, Inc., Chicago, Ill., USA).

\section{Results}

Fifty-four eligible participants $[61.5 \pm 8.2$ years old, body mass index (BMI) $34.6 \pm 4.3,77 \%$ male] were recruited. The average $\mathrm{HbA}_{1 \mathrm{C}}$ of participants was $7.7 \pm$ $1.5 \%$. All participants had a VPT score of $25 \mathrm{~V}$ or higher for at least one foot and the average VPT values were 56.3 $\pm 24 \mathrm{~V}$. 


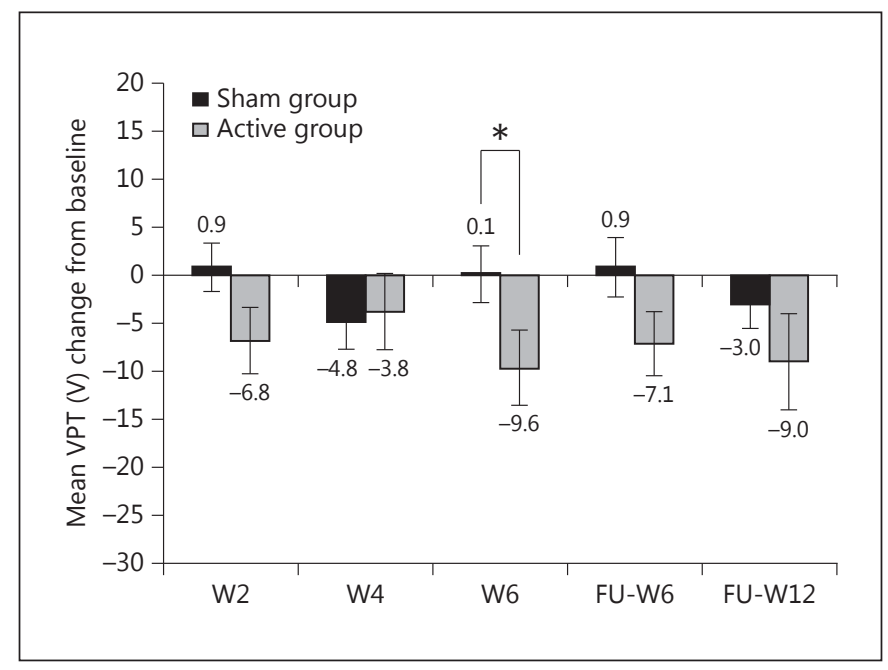

Fig. 2. Change in VPT from baseline. ${ }^{*}$ Significant difference $(\mathrm{p}<$ 0.05 ) between the groups. $\mathrm{W}=\mathrm{Week} ; \mathrm{FU}=$ follow-up.

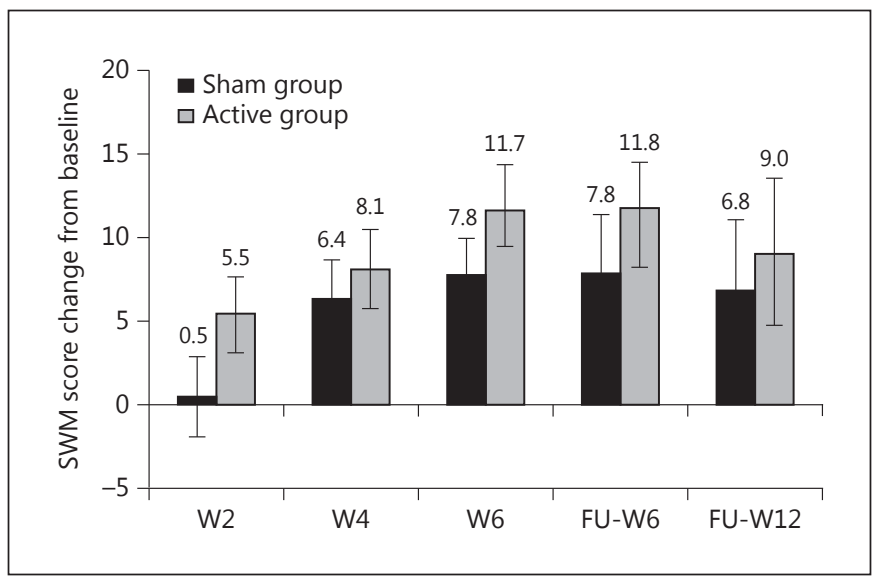

Fig. 4. Change in Semmes-Weinstein monofilament (SWM) scores. $\mathrm{W}=\mathrm{Week}$; $\mathrm{FU}$ = follow-up.

There was a significant interaction of treatment and visit for the VPT scores (fig. 2). At the week 6 treatment visit, the active group had a significantly improved score $(-9.6 \pm 15.9 \mathrm{~V})$ relative to baseline in comparison to the sham group $(0.1 \pm 19.5 \mathrm{~V})$. Although the active group maintained a greater reduction in VPT at follow-up, the difference between active and sham subjects was not significant at follow-up visits. The main effect of visit was significant for the monofilament data (fig. 3). Scores significantly improved at treatment weeks 4 and 6 as well as follow-up week 6 , relative to the treatment week 2 values.

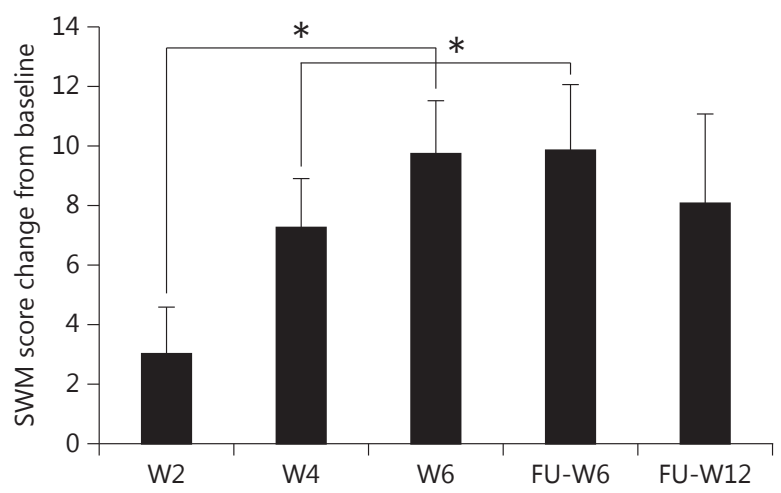

Fig. 3. Change in Semmes-Weinstein monofilament (SWM) scores from baseline. ${ }^{*}$ Monofilament scores were significantly higher at treatment weeks 4 and 6 as well as at the 6 -week follow-up in comparison to treatment week $2 . \mathrm{W}=\mathrm{Week} ; \mathrm{FU}=$ follow-up.

Although there was a trend of the active group displaying a greater improvement in monofilament scores, there was no significant difference between groups (fig. 4). Coefficients of variance ranged from 94 to $2,419 \%$ for the monofilament data.

A subgroup of 13 subjects was tested for postural control as a pilot study to explore the benefit of the proposed plantar stimulation for improving the balance. Table 1 summarizes the demographic information of participants. No statistically significant differences were observed between sham and active groups ( $p>0.05)$.

A significant correlation was observed between $\mathrm{HbA}_{1 \mathrm{C}}$ level and COM sway during the eyes-closed condition $(\mathrm{r}=0.63, \mathrm{p}=0.02$; fig. 5). On the same note, a negative correlation was observed between VPT value and COM sway during the eyes-closed condition $(\mathrm{r}=-0.47)$, but it was not significant in our sample size $(\mathrm{p}=0.1)$. No significant correlation was found between COM sway during eyes-closed and BMI as well as age of participants $(|\mathrm{r}|<0.4, \mathrm{p}>0.05)$. On the same note, no significant was found between COM sway during eyes-open and parameters of interest $(|\mathrm{r}|<0.2, \mathrm{p}>0.05)$, despite a relatively high correlation between body sway during the eyes-open and eyes-closed condition $(\mathrm{r}=0.75, \mathrm{p}=0.003)$.

At baseline, no significant difference was observed between the sham and active groups for $\mathrm{HbA}_{1 \mathrm{C}}$ level, BMI, age, and COM sway during both eyes-open and eyesclosed conditions. Since a few of the participants were unable to maintain balance during the eyes-closed condition 
5

Fig. 5. Correlation between $\mathrm{HbA}_{1 \mathrm{C}}$ level and COM sway (during eyes-closed) at baseline.

Fig. 6. Using subcutaneous electrical stimulation, postural control quantified by the area of COM sway (during eyes-open) was improved in the active group. * Significant improvement in postural control in the active group during treatment $(\mathrm{p}<0.05)$.

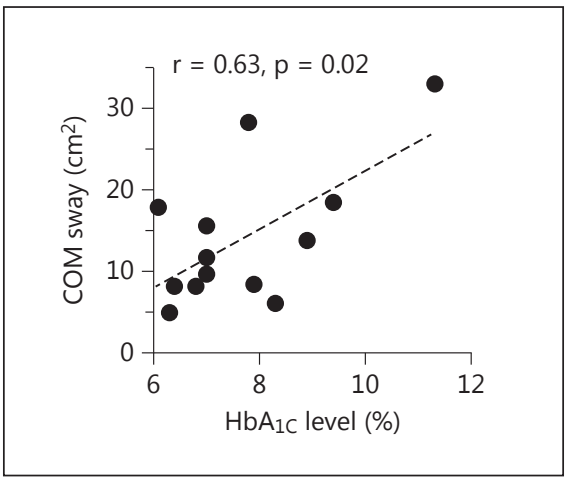

6

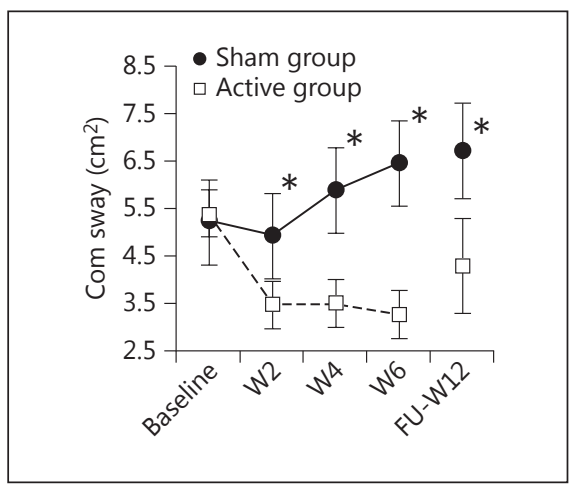

Table 1. Participants' demography

\begin{tabular}{|c|c|c|c|c|c|c|}
\hline & \multicolumn{3}{|l|}{ All subjects } & \multicolumn{3}{|c|}{ Subsample enrolled in the balance test } \\
\hline & active group & sham group & $\mathrm{p}$ value & active group & sham group & $\mathrm{p}$ value \\
\hline Patients, $\mathrm{n}$ & 25 & 29 & & 5 & 8 & \\
\hline Age, years & $61.6 \pm 8.3$ & $61.4 \pm 8.2$ & 0.92 & $59.0 \pm 6$ & $59.4 \pm 10$ & 0.86 \\
\hline $\mathrm{HbA}_{1 \mathrm{C}}, \%$ & $7.6 \pm 1.6$ & $7.1 \pm 1.5$ & 0.23 & $7.7 \pm 1.3$ & $7.7 \pm 1.6$ & 0.94 \\
\hline VPT, V & $46.8 \pm 23$ & $37.6 \pm 22$ & 0.13 & $56.8 \pm 15.9$ & $43.4 \pm 19.3$ & 0.07 \\
\hline
\end{tabular}

in some of the follow-up assessments, only the balance data during the eyes-open condition was compared between groups as well as compared to baseline. Results suggest a significant improvement in postural control in the active group during treatment ( $\mathrm{p}<0.05$; fig. 6). The area of COM sway was significantly reduced on average $36 \%$ at treatment week 2 compared to baseline ( $\mathrm{p}<0.003)$. Although an additional $18 \%$ reduction in COM sway was observed at week 6 compared to week 2, it was not significant ( $\mathrm{p}>$ $0.6)$. The overall improvement was retained at the end of study $(\mathrm{p}=0.5)$, despite a $38 \%$ nonsignificant increase $(\mathrm{p}=$ 0.07 ) in the COM sway. Interestingly, no significant difference was observed in the sham group during the study ( $p>0.7$ ). No related adverse events were reported.

\section{Discussion}

While low-level electrical stimulation has been used in practice for treating neuropathic pain [22, 23], we could not find support for its use for treating somatosensory loss in DPN. Low-level electrical stimulation is supported by a large body of basic science and clinical studies. The body of work in this area suggests opportunities for better patient care using a simple, inexpensive approach that has few adverse effects. As a potential aid in patients with loss of sensation, neurological low-level stimulation has been reported to improve the performance of the types of mechanoreceptor cells that provide protective sensation in the feet $[13,18]$. One of the theories of electrical stimulation benefits arises from the creation of background noise, or synaptic noise. Previous studies have found that the sensation threshold of tactile and proprioceptive systems is significantly lowered during low-level electrical stimulation [12-17]. The pathways of gait disturbance in DPN involve both the large and small nerve fibers. Schwann cells have been shown to decrease in diabetics; moreover, nerve function can be effected by hyperglycemic states, hypoxia, and other changes. Loss of Schwann cells interrupts the normal salutatory conduction of nerves and may lead to a decrease in nerve conduction. Using an animal model of nerve damage, Singh et al. [24] demonstrated that electrical stimulation benefits Schwann cell regeneration and may serve as a tool for addressing the changes in the nerve conduction pathway in diabetics. However, the animal model used in their study may vary from a 'diabetic model' of nerve damage and thus additional study is required to confirm their observation in a diabetic model of nerve damage as well as in human. 
Another theory describing the benefits of electrical stimulation in recovery of nerve damage is the potential benefit of electrical stimulation in improving skin perfusion. Microvascular damage has been shown to be a contributing factor in the pathology of peripheral neuropathy [25]. Diminished local blood flow can initiate oxidative stress and the release of factors that impede the normal passage of neurological signals. One study showed an increase in capillary basement membrane $(\mathrm{p}<0.002)$, endothelial cells $(\mathrm{p}<0.003)$, total diffusion barrier $(\mathrm{p}<$ $0.001)$ and a reduction in oxygen diffusion capacity $(\mathrm{p}<$ 0.001) in diabetics compared to control subjects [26]. Electrical stimulation has been shown to increase dermal perfusion [29-31], possibly through a release of vasoendothelial growth factor (VEGF) $[32,33]$. The increase in VEGF may counter the pathways to DPN through stimulation of angiogenesis for increase perfusion of endoneurial microvessels. Additionally, VEGF has been shown to induce Schwann cell proliferation, stimulate axonal outgrowth and promote survival of neurons and Schwann cells in cultured animal cells. VEGF concentrations of 30, 50 and $100 \mathrm{ng} / \mathrm{ml}$ showed significant axonal outgrowth after $48 \mathrm{~h}(\mathrm{p}<0.05)$ [34]. Schratzberger et al. [35] demonstrated functional VEGF receptors on Schwann cells and proposed VEGF has a direct effect on the cellular function. They showed VEGF has a dose-dependent stimulation on Schwann cell migration from 10 to $500 \mathrm{ng} /$ $\mathrm{ml}$. In vitro studies showed electrical stimulation increases VEGF secretion [32] and increased mRNA activity in skeletal muscles [33].

Diabetics with peripheral neuropathy frequently suffer from postural instability that can lead to falls, fractures, depression, anxiety and decreased quality of life $[3-5,37,38]$. The loss of planter cutaneous sensation is believed to responsible for moment disturbances in peripheral neuropathy. Meyer et al. [38] anesthetized the cutaneous planter sole of healthy subjects, the resulting reduced planter sensitivity showed redistribution of corrective torque from ankle and trunk to the hip joint. Subjects adopted an abnormal postural strategy which may increase difficulty of regaining balance for fall prevention the diabetic population due to body girth or amputations. As peripheral neuropathy results in loss of ankle, knee and hip proprioception, postural stability and gait may be more pronounced [38]. In addition, loss of proprioception could alter the ability of subject in obstacle negotiation, another factor in increasing risk of falling in individuals suffering from DPN [6]. Grewal et al. [6] demonstrated that neuropathy severity quantified by VPT has a relatively high correlation with ability of older adults in perception of lower extremity position, which in turn impacts their ability in successful obstacle clearance. Early correction of gait may prevent secondary injuries from abnormal gait or prolonged weight shifting. The protective sensation assessments of DPN indicated that the aqueous electrical stimulation therapy provided in this study provided some benefit to subjects. This is the first known study to demonstrate a therapeutic effect from electrical stimulation that persisted after administration of treatment. Other studies have focused on assessing patients while they actively received stimulation. This is important in considering widespread use of the therapy. With the use of the aqueous electrical stimulation therapy, subjects were able to go about their normal daily activities without being encumbered by the device.

Although the significant difference in VPT observed at the end of treatment did not remain statistically different at follow-up, there was a continued trend. It is possible that in order to maintain a benefit, active treatment must continue at fixed intervals following an initial 6-week program. The finding of a significant main effect of visit for the monofilament data was not expected. Although it was expected that the active treatment subjects would see an improvement in monofilament detections, the sham treatment subjects were not expected to improve. The fact that both groups improved may be indicative of a learning or practice effect associated with doing the monofilament tests numerous times. While the active subjects had better monofilament scores at each week than the sham subjects, the difference never reached statistical significance. The large variability between subjects was an important factor in the lack of a statistical difference. One potential contributor to the large variance between subjects for both monofilament and VPT data is the somewhat subjective nature of the tests that are dependent on subjects' recognition and verbalization of stimuli.

By not requiring verbalized responses, the postural control assessments could be considered to be less subjective in nature. In this study we observed a significant improvement in postural sway in the active group. These findings are in line with those of Gravelle et al. [14] that center of pressure sway parameters decreased when lowlevel electrical noise was applied to older adult's knees. This randomized pilot study provides essential preliminary data on the ability of electrical stimulation provided via an aqueous solution to improve postural stability in individuals with DPN. Additionally, this study revealed an association between poor hyperglycemia control and postural instability. 
Our finding of the association of improved glycemic control and less postural sway (fig. 5) merits further discussion. Intensive glycemic control in the elderly is a controversial topic for clinicians trying to balance the risks of future diabetes complication with hypoglycemic events and possible cardiovascular events in those with preexisting cardiovascular disease [39]. Recently, Petrofsky et al. [40] studied 37 subjects' postural sway and cortical activity using electroencephalography during a variety of balance tasks. They found elderly subjects had increased cortical activity during complicated balance tasks versus younger subjects where this activity was likely addressed by the lower brain in the vestibular and cerebellar areas. They also found that diabetes subjects had higher cortical activity over older subjects. Diabetes subjects have also been described to have impaired cognition that may be more related to hypoglycemia and possibly the cumulative number of hypoglycemia events [41]. Impaired cognition with increased cortical demands from decreased sensory, vestibular, and visual input may explain our finding of improved glucose control and postural sway.

There are limitations to our study. We may have been underpowered to detect changes in the balance parameters or for those patients with an asymmetric presentation. Further studies with larger sample sizes are required to confirm these results and address potential changes in other balance parameters (e.g. postural coordination). In addition, some of the participants, particularly in the sham group, failed to maintain balance during the eyesclosed condition in some of the follow-up assessments. Thus, we were not able to explore a potential benefit of the proposed intervention in improvement balance during the eyes-closed condition. Another study design with a shorter period of balance assessment during the eyesclosed condition (e.g. $10 \mathrm{~s}$ instead of $30 \mathrm{~s}$ ) may address this challenge. Additionally, another study design should be addressed to explore the benefit of this technique in improving gait, reducing risk of falling, and preventing lower extremity problems (e.g. diabetic foot ulcer) due to peripheral neuropathy.

\section{Conclusion}

Many patients with DPN experience a marked loss of mechanical sensitivity, a condition that puts them at risk for the formation of dangerous skin ulcers and postural instability. This randomized pilot study provides essential preliminary data on the ability of low-level electrical stimulation provided via an aqueous solution to improve sensory function and postural stability in individuals with DPN. Additionally, it suggests that diabetic patients with poor hyperglycemic control may be at higher risk of falling and therefore require further attention for preventing fall accidents.

\section{Acknowledgements}

This study was partially supported by WaveRx Incorporated funding. The authors would like to thank Dr. Lee Rogers for assisting in patient recruitment and data collection.

\section{Disclosure Statement}

The authors have no conflicts of interest to disclose.

\section{References}

1 Corriveau H, Prince F, Hebert R, Raiche M, Tessier D, Maheux P, Ardilouze JL: Evaluation of postural stability in elderly with diabetic neuropathy. Diabetes Care 2000;23: 1187-1191.

2 National Diabetes Statistics. http://diabetes. niddk.nih.gov/dm/pubs/statistics/\#fast (accessed February 2011).

-3 Wrobel JS, Najafi B: Diabetic foot biomechanics and gait dysfunction. J Diabetes Sci Technol 2010;4:833-845.

4 Najafi B, Horn D, Marclay S, Crews RT, Wu S, Wrobel JS: Assessing postural control and postural control strategy in diabetes patients using innovative and wearable technology. J Diabetes Sci Technol 2010;4:780-791.
5 Najafi B, Bharara M, Talal T, Armstrong DG: Advances in balance assessment and balance training for diabetes. Diabetes Manag 2012;2: 293-308.

6 Grewal G, Sayeed R, Yeschek S, Menzies RA, Talal TK, Lavery LA, Armstrong DG, Najafi B: Virtualizing the assessment: a novel pragmatic paradigm to evaluate lower extremity joint perception in diabetes. Gerontology 2012;58:463-471.

7 Schwartz AV, Hillier TA, Sellmeyer DE, Resnick HE, Gregg E, Ensrud KE, Schreiner PJ, Margolis KL, Cauley JA, Nevitt MC, Black DM, Cummings SR: Older women with diabetes have a higher risk of falls: a prospective study. Diabetes Care 2002;25:1749-1754.
8 Volpato S, Leveille SG, Blaum C, Fried LP, Guralnik JM: Risk factors for falls in older disabled women with diabetes: the women's health and aging study. J Gerontol A Biol Sci Med Sci 2005;60:1539-1545.

$\checkmark 9$ Wallace C, Reiber GE, LeMaster J, Smith DG, Sullivan K, Hayes S, Vath C: Incidence of falls, risk factors for falls, and fall-related fractures in individuals with diabetes and a prior foot ulcer. Diabetes Care 2002;25:1983-1986.

10 Wrobel JS, Crews RT, Connolly JE: Clinical factors associated with a conservative gait pattern in older male veterans with diabetes. J Foot Ankle Res 2009;2:11.
Electrical Stimulation Therapy for Improving DPN 
11 CDC: Lower extremity disease among persons aged $\geq 40$ years with and without diabetes - United States, 1999-2002. Atlanta, CDC, 2005.

$\checkmark 12$ Dhruv NT, Niemi JB, Harry JD, Lipsitz LA, Collins JJ: Enhancing tactile sensation in older adults with electrical noise stimulation. Neuroreport 2002;13:597-600.

13 Priplata AA, Patritti BL, Niemi JB, Hughes R, Gravelle DC, Lipsitz LA, Veves A, Stein J, Bonato P, Collins JJ: Noise-enhanced balance control in patients with diabetes and patients with stroke. Ann Neurol 2006;59:4-12.

14 Gravelle DC, Laughton CA, Dhruv NT, Katdare KD, Niemi JB, Lipsitz LA, Collins JJ: Noise-enhanced balance control in older adults. Neuroreport 2002;13:1853-1856.

15 Reed BV: Effect of high-voltage pulsed electrical stimulation on microvascular permeability to plasma proteins. A possible mechanism in minimizing edema. Phys Ther 1988;68: 491-495.

16 Reich JD, Cazzaniga AL, Mertz PM, Kerdel FA, Eaglstein WH: The effect of electrical stimulation on the number of mast cells in healing wounds. J Am Acad Dermatol 1991; 25:40-46.

17 Najafi B, Crews R, Wrobel JS: A novel plantar stimulation technology for improving postural control in patients with diabetic peripheral neuropathy - a double-blinded, randomized study. 6th International Symposium on the Diabetic Foot, Noordwijkerhout, The Netherlands, 2011.

$\checkmark 18$ Khaodhiar L, Niemi JB, Earnest R, Lima C, Harry JD, Veves A: Enhancing sensation in diabetic neuropathic foot with mechanical noise. Diabetes Care 2003;26:3280-3283.

$\checkmark 19$ Boulton AJ, Armstrong DG, Albert SF, Frykberg RG, Hellman R, Kirkman MS, Lavery LA, Lemaster JW, Mills JL Sr, Mueller MJ, Sheehan P, Wukich DK: Comprehensive foot examination and risk assessment: a report of the task force of the foot care interest group of the American Diabetes Association, with endorsement by the American Association of Clinical Endocrinologists. Diabetes Care 2008;31:1679-1685.

20 Lavery LA, Murdoch DP, Williams J, Lavery DC: Does anodyne light therapy improve peripheral neuropathy in diabetes? A doubleblind, sham-controlled, randomized trial to evaluate monochromatic infrared photoenergy. Diabetes Care 2008;31:316-321.
21 Armstrong DG, Hussain SK, Middleton J, Peters EJ, Wunderlich RP, Lavery LA: Vibration perception threshold: are multiple sites of testing superior to single-site testing on diabetic foot examination? Ostomy Wound Manage 1998;44:70-74, 76.

22 Al-Jehani H, Jacques L: Peripheral nerve stimulation for chronic neurogenic pain. Prog Neurol Surg 2011;24:27-40.

23 Baheti DK: Neuropathic pain - recent trends in management. J Indian Med Assoc 2001;99: 692, 694-695, 697.

24 Singh B, Xu QG, Franz CK, Zhang R, Dalton C, Gordon T, Verge VM, Midha R, Zochodne DW: Accelerated axon outgrowth, guidance, and target reinnervation across nerve transection gaps following a brief electrical stimulation paradigm. J Neurosurg 2012;116:498512.

25 Duby JJ, Campbell RK, Setter SM, White JR, Rasmussen KA: Diabetic neuropathy: an intensive review. Am J Health Syst Pharm 2004; 61:160-173; quiz 175-176.

26 Malik RA, Newrick PG, Sharma AK, A Jennings, Ah-See AK, Mayhew TM, Jakubowski J, Boulton AJ, Ward JD: Microangiopathy in human diabetic neuropathy: relationship between capillary abnormalities and the severity of neuropathy. Diabetologia 1989;32:92-102.

27 Forst T, Pfutzner A, Bauersachs R, Arin M Bach B, Biehlmaier H, Kustner E, Beyer J: Comparison of the microvascular response to transcutaneous electrical nerve stimulation and postocclusive ischemia in the diabetic foot. J Diabetes Complications 1997;11:291297.

28 Gilcreast DM, Stotts NA, Froelicher ES, Baker LL, Moss KM: Effect of electrical stimulation on foot skin perfusion in persons with or at risk for diabetic foot ulcers. Wound Repair Regen 1998;6:434-441.

29 Peters EJ, Armstrong DG, Wunderlich RP, Bosma J, Stacpoole-Shea S, Lavery LA: The benefit of electrical stimulation to enhance perfusion in persons with diabetes mellitus. J Foot Ankle Surg 1998;37:396-400; discussion 447-448.

-30 Clover AJ, McCarthy MJ, Hodgkinson K, Bell PR, Brindle NP: Noninvasive augmentation of microvessel number in patients with peripheral vascular disease. J Vasc Surg 2003;38: 1309-1312.

31 Cramp AF, Gilsenan C, Lowe AS, Walsh DM: The effect of high- and low-frequency transcutaneous electrical nerve stimulation upon cutaneous blood flow and skin temperature in healthy subjects. Clin Physiol 2000;20:150157.
2 Zhao M, Bai H, Wang E, Forrester JV, McCaig $\mathrm{CD}$ : Electrical stimulation directly induces pre-angiogenic responses in vascular endothelial cells by signaling through VEGF receptors. J Cell Sci 2004;117:397-405.

-33 Kanno S, Oda N, Abe M, Saito S, Hori K, Handa Y, Tabayashi K, Sato Y: Establishment of a simple and practical procedure applicable to therapeutic angiogenesis. Circulation 1999; 99:2682-2687.

34 Sondell M, Lundborg G, Kanje M: Vascular endothelial growth factor has neurotrophic activity and stimulates axonal outgrowth, enhancing cell survival and Schwann cell proliferation in the peripheral nervous system. J Neurosci 1999; 19:5731-5740.

- 35 Schratzberger P, Schratzberger G, Silver M, Curry C, Kearney M, Magner M, Alroy J, Adelman LS, Weinberg DH, Ropper AH, Isner JM: Favorable effect of VEGF gene transfer on ischemic peripheral neuropathy. Nat Med 2000;6:405-413.

36 Najafi B, Khan T, Fleischer A, Wrobel J: The impact of footwear and walking distance on gait stability in diabetic patients with peripheral neuropathy. J Am Podiatr Med Assoc. 2013;103:165-173.

37 Vileikyte L, Peyrot M, Gonzalez JS, Rubin RR, Garrow AP, Stickings D, Waterman C, Ulbrecht JS, Cavanagh PR, Boulton AJ: Predictors of depressive symptoms in persons with diabetic peripheral neuropathy: a longitudinal study. Diabetologia 2009;52:1265-1273.

38 Meyer PF, Oddsson LI, De Luca CJ: Reduced plantar sensitivity alters postural responses to lateral perturbations of balance. Exp Brain Res 2004;157:526-536.

39 Dailey G: Early and intensive therapy for management of hyperglycemia and cardiovascular risk factors in patients with type 2 diabetes. Clin Ther 2011;33:665-678.

40 Petrofsky JS, Alshammari F, Lee H, Yim JE, Bains G, Khowailed IA, Deshpande PP, Potnis P, Tse F, Cavalcanti P: Electroencephalography to assess motor control during balance tasks in people with diabetes. Diabetes Technol Ther 2012;14:1068-1076.

41 Brismar T: The human EEG - physiological and clinical studies. Physiol Behav 2007;92: 141-147. 